\title{
Composição de plantas invasoras em diferentes sistemas integrados de produção agropecuária
}

\author{
Daniel de Queiroz Barbosa', Anibal de Moraes², Claudete Reisdorfer Lang ${ }^{2}$, Leandro de Oliveira Bittencourt², \\ João Pedro Tavares Fernandes ${ }^{3}$ e Camila Crestani ${ }^{4}$
}

\begin{abstract}
Resumo - No âmbito global se reconhece o potencial dos sistemas integrados como uma via sustentável para alimentar nove bilhões de pessoas em 2050. Esses sistemas são capazes de incrementar a resiliência ambiental pelo aumento da diversidade biológica e pela efetiva e eficiente ciclagem de nutrientes, o que acarreta a melhoria da qualidade do solo, além de prover serviços ecossistêmicos e contribuir para a mitigação das mudanças climáticas. O objetivo do trabalho foi avaliar a ocorrência e a composição da comunidade de plantas daninhas na cultura do milho em Sistemas Integrados de Produção Agropecuária (SIPAs). O delineamento experimental foi o de blocos casualizados (DBC), com 4 tratamentos e 3 repetições. A variedade de milho BRS Sol da Manhã foi cultivada em quatro sistemas: lavoura (L), integrado com lavoura-pecuária (LP), lavoura-floresta (LF) e lavoura-pecuária-floresta (LPF), todos sem a utilização de herbicida. Foram avaliadas a ocorrência e a composição de plantas daninhas nos diferentes sistemas de produção por meio de um levantamento da composição botânica em matéria seca $\left(\mathrm{kg} \mathrm{ha}^{-1}\right)$. Verificou-se grande contribuição de Megathyrsus maximus cv. Áries em todos os sistemas, principalmente no LPF, assim como o predomínio de Urochloa brizantha no LP, além do predomínio de Hemarthria altíssima no sistema LF. O SIPA demonstrou ser capaz de produzir $5,8 \mathrm{t} \mathrm{ha} \mathrm{a}^{-1}$ milho sem uso de herbicidas.
\end{abstract}

Termos para indexação: Matologia; Planta daninha; Botanal.

\section{Composition of weeds in different integrated crop-livestock system}

Abstract - Globally, the potential of integrated systems is recognized as a sustainable way to feed nine billion people in 2050. These systems can increase environmental resilience by increasing biological diversity and by the effective and efficient cycling of nutrients, which entails improving soil quality, in addition to providing ecosystem services and contributing to climate change adaptation and mitigation. The objective of this work was to evaluate the occurrence and species composition of weed in the corn crop in the Integrated Crop-Livestock System (ICLS). The experimental design was randomized blocks, with 4 treatments and 3 repetitions. The variety of "BRS Sol da Manhã" corn was cultivated in four systems: crop (C), integrated with crop-livestock (CP), crops-forest (CF) and crops-livestock-forest (CLF), all without the use of herbicide. The occurrence and composition of weeds in the different production systems was evaluated through a survey of the botanical composition in dry matter ( $\left.\mathrm{kg} \mathrm{ha}^{-1}\right)$. There was a large contribution of Megathyrsus maximus $\mathrm{cv}$. Áries in all systems, mainly in the CLF, as well as a predominance of Urochloa brizantha in the $\mathrm{CL}$, in addition to the of very high contribution Hemarthria altissima in the $\mathrm{CF}$ system. The ICLS produced $5.8 \mathrm{t} \mathrm{ha}^{-1}$ corn without the use of herbicides.

Index terms: Herbology; Weed; Botanal.

Plantas que causam danos às atividades e à saúde humana e ao meio ambiente têm designações compatíveis com sua função biológica: planta parasita, planta exótica invasora, planta pioneira, planta trepadeira, entre outras. Segundo Pitelli (2015), "planta daninha" é um termo genérico e impreciso, porque não se refere a qualquer função biológica, sendo mais apropriado o termo "planta invasora", em razão da infestação de áreas de ação antrópica.
As plantas invasoras são um dos grandes desafios da agricultura, pois podem reduzir a produtividade e a qualidade das culturas de interesse econômico. Nesse aspecto os Sistemas Integrados de Produção Agropecuária (SIPAs) são uma alternativa para auxiliar no controle dessas plantas devido à diversidade e à complexidade empregada neles (SCHUSTER et al., 2019) 5 .

Os SIPAs têm se mostrado como opção viável de produção, pois promo- vem a melhoria da qualidade do solo, são mais eficientes no uso dos recursos naturais e reduzem custos de produção, mantendo níveis de produtividade elevados. Além disso, são eficientes na recuperação de áreas degradadas (CARVALHO et al., 2014) e surgem como alternativa à ativação econômica de Áreas de Proteção Ambiental (APAs).

A maioria dos cultivos agrícolas tem como base o uso intensivo de herbicidas, resultando em um aumento ex-

Recebido em 4/8/2021. Aceito para publicação em 12/11/2021.

http://dx.doi.org/10.52945/rac.v34i3.1274

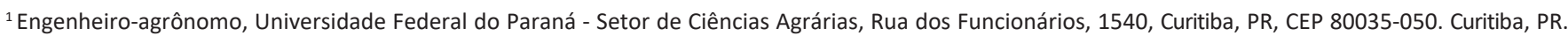
e-mail: danielqbarbosa@gmail.com.

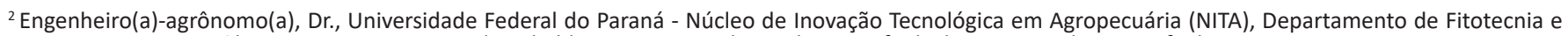
Fitossanitarismo, Curiti ba, PR, 80035-050. e-mail: anibaldemoraes@gmail.com, langc@ufpr.br, bitt encourtoliveira@ufpr.br .

${ }^{3}$ Engenheiro-agrônomo, Doutorando, UFPR - Programa de Pós-Graduação em Agronomia - Produção Vegetal. e-mail: joaopedroagronomo@gmail.com.

${ }^{4}$ Engenheira-agrônoma, Mestranda, UFPR - Programa de Pós-Graduação em Agronomia - Produção Vegetal. e-mail: camilacrestani2015@gmail.com.

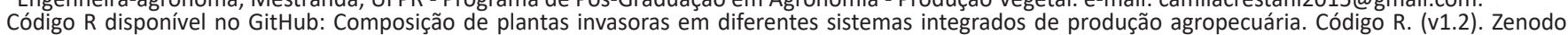
DOI:10.5281/zenodo.5570497 
ponencial no número de genótipos de plantas resistentes (EMBRAPA, 2006). Porém, em algumas áreas de proteção ambiental, fica vedada a utilização de agroquímicos (PIASSETTA, 2021), sendo portanto necessárias outras estratégias de controle.

Uma alternativa para manejo de plantas invasoras consiste no emprego de práticas conservacionistas, como o plantio direto, que modifica a dinâmica do ambiente, alterando a incidência de plantas daninhas. Isso se deve à barreira física gerada pela palhada no solo, que impede o fluxo de emergência da comunidade de plantas infestantes (DOMINSCHEK et al., 2021), e também devido à liberação de substâncias alelopáticas que alteram a dinâmica e germinação do banco de sementes (SCHUSTER et al., 2019).

Outra prática que pode ser adotada é a rotação de culturas. Ao incluir pastagens bem manejadas no sistema de rotação, ocorre a redução da incidência de plantas invasoras (SCHUSTER et al., 2019), já que o fechamento do dossel reduz a quantidade de luz e torna o ambiente menos propício ao desenvolvimento de plantas. Assim, quanto mais diversas as rotações, mais eficientes serão os efeitos de supressão (WEISBERGER et al., 2019).

A população de plantas invasoras não provoca impacto significativo na produtividade de milho em diferentes arranjos de SIPA. Partindo-se dessa hipótese, avaliou-se a composição de plantas invasoras na cultura do milho integrada com eucaliptos, nos seguintes casos: rotação com pastagem, em área de eucalipto com alternância de pastagem e cultivo na entrelinha, comparados com monocultivo de milho.

O experimento foi conduzido no Núcleo de Inovação Tecnológica em Agropecuária (NITA), da Estação Experimental do Canguiri, pertencente à Universidade Federal do Paraná, no município de Pinhais, PR ( $25^{\circ} 23^{\prime} 30^{\prime \prime}$ S latitude, 4907'30"W longitude, $935 \mathrm{~m}$ altitude). Precipitação anual de $1.400 \mathrm{~mm}$, temperatura média mínima e máxima de $12,5^{\circ} \mathrm{C}$ e $22,5^{\circ} \mathrm{C}$, respectivamente, sujeita a geadas frequentes e severas (IDRPARANÁ, 2019). O solo é classificado como Cambissolo Háplico Tb distrófico típico (EMBRAPA, 2018).
Os tratamentos foram compostos pelos sistemas de produção LavouraPecuária (LP), Lavoura-Floresta (LF) e Lavoura-Pecuária-Floresta (LPF), comparando-os com o monocultivo tradicional de milho (L). Os sistemas de L e LF foram semeados com milho (Zea mays L.) no verão e aveia-preta ( $A$. strigosa) no inverno para cobertura do solo. As áreas de LP e LPF foram pastejadas durante três anos, utilizando como pastagem de inverno a aveia-preta ( $A$. strigosa) e como pastagem de verão o capim Aries (M. maximus). No inverno anterior à semeadura do milho não há pastejo, sendo as áreas semeadas com aveia-preta para cobertura do solo. Os tratamentos foram instalados num delineamento experimental de blocos casualizados (DBC) com três repetições em 2013. A avaliação de plantas invasoras foi conduzida entre janeiro e abril de 2021, sendo o primeiro ano de avaliação.

Os teores de nutrientes identificados pela análise de solo são apresentados na Tabela 1, embora na área experimental seja realizada adubação de sistemas, levando em consideração a sinergia da relação solo-planta-animal na ciclagem de nutrientes e a liberação de $P$ retido na palhada, induzida pela adubação de $\mathrm{N}$ na semeadura (LEVINSKI-HUF, 2018). A área foi adubada com $60 \mathrm{~kg} \mathrm{ha}^{-1}$ de ureia na semeadura do milho sobre palhada verde (DOMINSCHEK, 2018). No estádio V4 do milho foram aplicados $250 \mathrm{~kg} \mathrm{ha}^{-1}$ de $\mathrm{KCl}, 200 \mathrm{~kg} \mathrm{ha}^{-1}$ de $\mathrm{P}$ e $300 \mathrm{~kg} \mathrm{ha}^{-1} \mathrm{de}$ ureia.

Foi realizado um levantamento da composição botânica em matéria seca ( $\left.\mathrm{kg} \mathrm{ha}^{-1}\right)$ das plantas invasoras que apareceram na entrelinha do milho nos estádios R5 e R6. A estimativa de participação na composição botânica foi adaptada a partir do método de Coser (1991), com quadrados de $1,0 \mathrm{~m}^{2}(1,0 \mathrm{x}$ $1,0 \mathrm{~m})$, usando-se o método da Massa Seca Ordenada de acordo com o mé-

todo BOTANAL (HARGREAVES \& KERR, 1992).

A partir disso, três quadrados de cada padrão (de 1 a 5) foram estimados, cortados e posteriormente secos em estufa a $65^{\circ} \mathrm{C}$, por 72 horas, para obtenção das medidas de massa observadas. Assim foi estimada uma equação de regressão da produção de matéria seca em função dos padrões estimados visualmente.

Foram coletadas 6 amostras, com 3 repetições (18 por tratamento), totalizando 72 amostras. As amostras foram convertidas, a partir da equação de regressão, na estimativa de matéria seca por hectare.

A produtividade de grãos do milho foi estimada através da determinação dos componentes de rendimento das plantas, exigindo a determinação da umidade e do peso médio dos grãos, espaçamento entre linhas e o número médio de espigas obtidos em várias amostragens. A amostragem foi realizada em 3 pontos por tratamento, selecionando aleatoriamente fileiras de 16 metros lineares por ponto.

As espigas foram debulhadas e os grãos pesados para obtenção da massa total úmida, e posterior correção da umidade para $15,5 \%$. A produtividade foi calculada pela expressão PROD $=[10.000 \times($ NExP $) / E M] / 1.000$, Onde PROD: produtividade (Kg ha $\left.{ }^{-1}\right)$; NE: número médio de espigas em 16 metros lineares; $\mathrm{P}$ : peso médio de grãos por espiga; EM: espaçamento médio entre linhas (RODRIGUES, 2005).

Utilizando software $\mathrm{R}$ e RStudio, foi realizada análise multivariada de ordenação pelos componentes principais (PCA), tendo a distância euclidiana como medida de similaridade entre os tratamentos.

Foram amostradas 8 espécies no total dos sistemas (Tabela 2). As variações $(53,1 \%)$ da contribuição da massa de

Tabela 1. Teores de nutrientes da área experimental Table 1. Nutrient content of the experimental area

\begin{tabular}{lccccccccc}
\hline & $\mathrm{pH}$ & $\mathrm{M} . \mathrm{O}$ & $\mathrm{P}$ & $\mathrm{K}$ & $\mathrm{Ca}$ & $\mathrm{Mg}$ & $\mathrm{Al}$ & $\mathrm{CTC}$ & $\mathrm{V}$ \\
\hline & $\mathrm{CaCl}^{2}$ & $\%$ & & & $\mathrm{Cmol} \mathrm{dm}^{-3}$ & & & $(\%)$ \\
\hline Testemunha & 5,28 & 3,53 & 2,81 & 0,16 & 5,1 & 3,6 & 0 & 10,32 & 68,90 \\
\hline Teor & Alto & $\begin{array}{c}\text { Muito } \\
\text { alto }\end{array}$ & Baixo & Médio & Alto & $\begin{array}{c}\text { Muito } \\
\text { alto }\end{array}$ & $\begin{array}{c}\text { Muito } \\
\text { baixo }\end{array}$ & $\begin{array}{c}\text { Muito } \\
\text { alto }\end{array}$ & Alto \\
\hline
\end{tabular}


plantas invasoras foram explicadas principalmente pelas espécies $M$. maximus e H. altíssima (Figura 1). Os sistemas LPF apresentaram maior relação com a espécie $M$. maximus e o sistema LF maior relação com $H$. altíssima em apenas um dos blocos.

Os vetores na Figura 2 representam a distribuição das espécies invasoras entre os tratamentos. O comprimento do vetor indica a variância da massa, ou seja, quanto maior o vetor, maior a contribuição para a composição de massa seca. $\mathrm{O}$ ângulo formado entre dois vetores indica o grau de correlação ou covariância entre as espécies. Um ângulo de $90^{\circ}$ indica ausência de correlação e um ângulo maior que $90^{\circ}$ indica correlação negativa ou inversa.

Verificou-se grande contribuição de $M$. maximus em todos os sistemas, principalmente no LPF, assim como o predomínio de $U$. brizantha no LP e ainda o sistema LF com predomínio de H. altíssima. Em contrapartida, onde há maior ocorrência destas gramíneas não há contribuição significativa de outras plantas invasoras, provavelmente porque elas não superaram a cobertura de solo (derivada do sistema de plantio direto) ou ainda porque ocorreu possivelmente efeito alelopático entre as espécies inventariadas.

A produtividade média de grãos de milho foi de $5.808 \mathrm{~kg} \mathrm{ha}^{-1}$ similar entre os sistemas de produção $(P>0,05)$, acompanhando a média de produtividade do milho safrinha no estado do Paraná (CONAB, 2021). Nas condições experimentais a produtividade do milho foi semelhante e se manteve na média estadual dos últimos dez anos, apesar da competição de plantas invasoras e sem o uso de herbicidas. Não houve homogeneidade na composição de plantas invasoras entre os tratamentos.

\section{Referências}

CARVALHO, P. C. D. F., MORAES, A. D., PONTES, L. D. S., ANGHINONI, I., SULC, R. M., BATELLO, C. Definições e terminologias para sistema integrado de produção agropecuária. Revista Ciência Agronômica, v.45, p.1040-1046, 2014. DOI: https://doi. org/10.1590/S1806-66902014000500020.

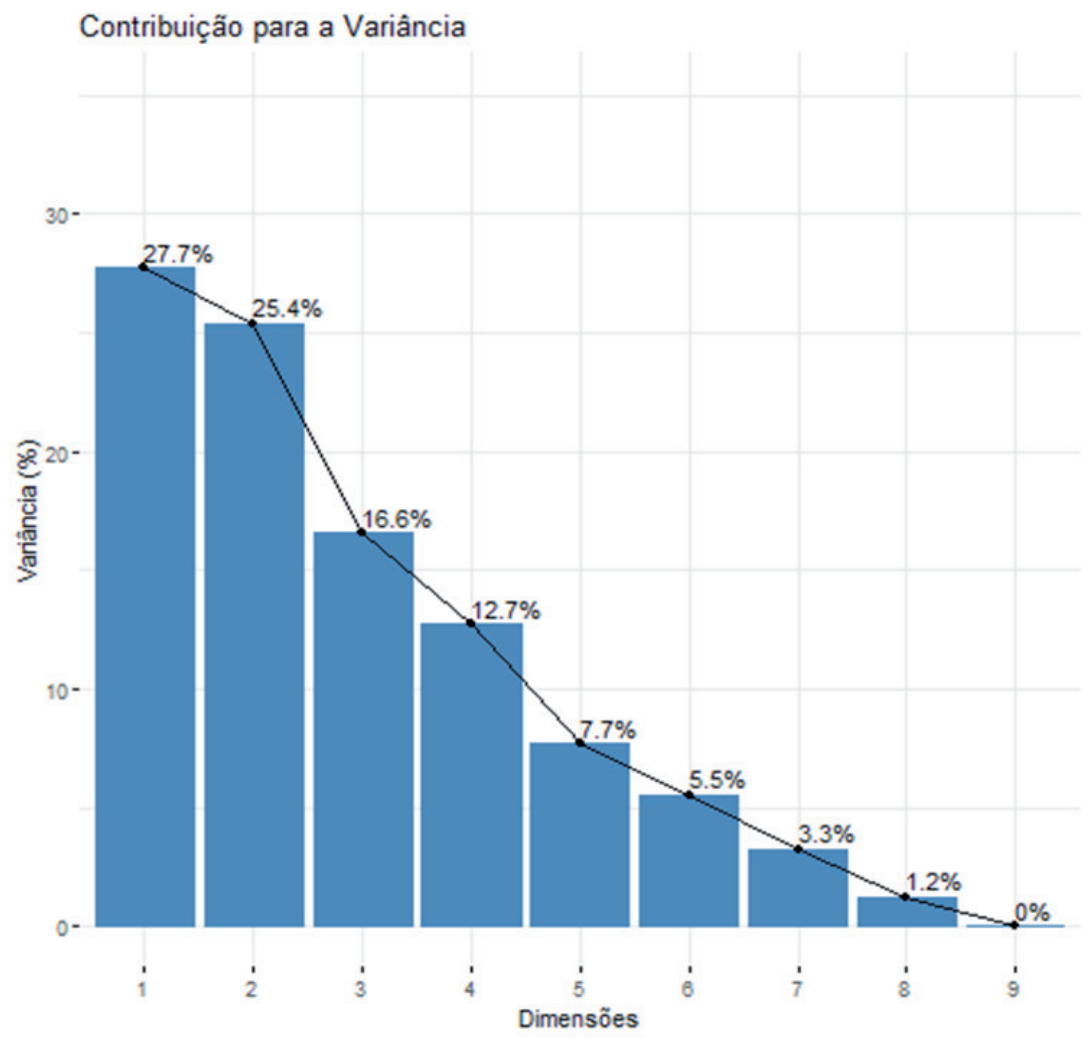

Figura 1. Contribuição de cada componente principal (massas de plantas invasoras) para a variância dos tratamentos

Figure 1. Contribution of each main component (mass of weed) to the variance of treatments

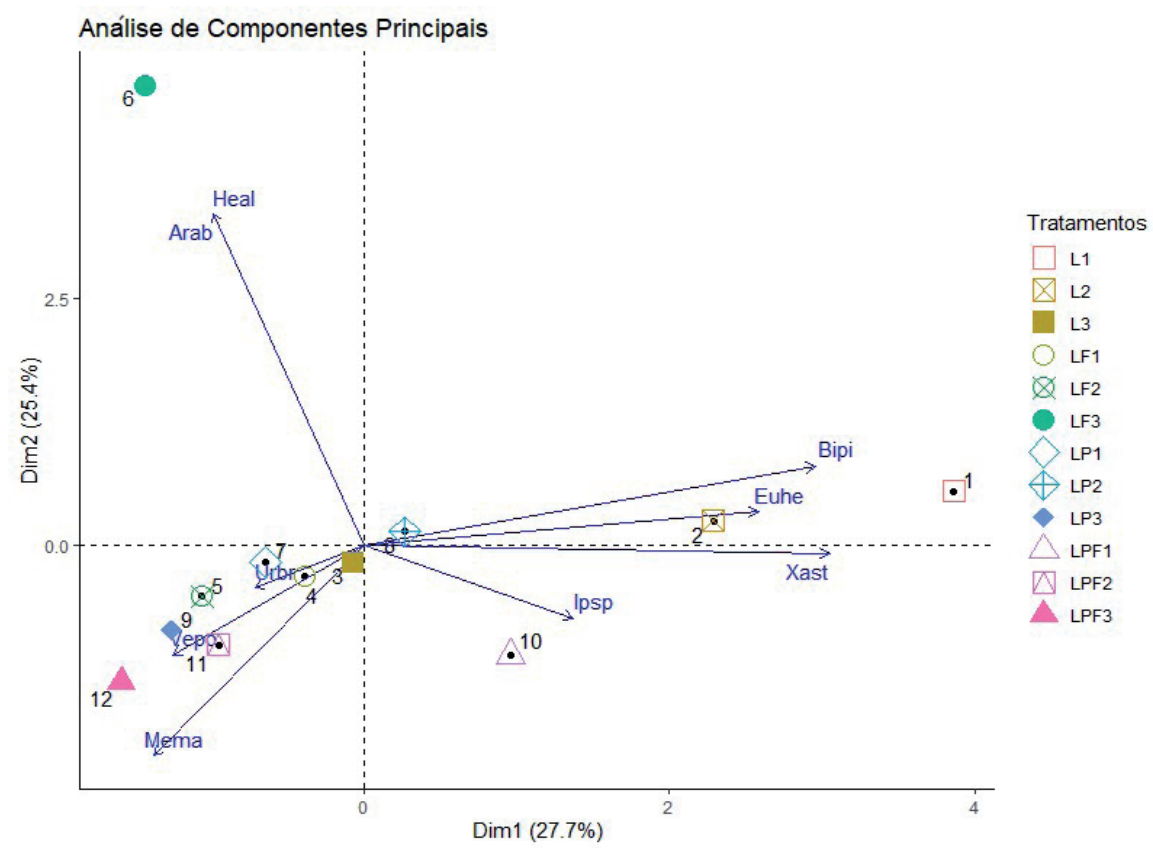

Figura 2. Ordenação dos Sistemas de Produção em função das massas das espécies invasoras M. maximus (Mema), Ipomoea sp. (Ipsp), V. polysphaera (Vepo), X. strumarium (Xast), B. pilosa (Bipi), U. brizantha (Urbr), H. altíssima (Heal), E. heterophylla (Euhe) pelos componentes principais

Figure 2. Ordering of Production Systems as a function of the mass of invasive species M. maximus (Mema), Ipomoea sp. (Ipsp), V. polysphaera (Vepo), X. strumarium (Xast), B. pilosa (Bipi), U. brizantha (Urbr), H. altissima (Heal), E. heterophylla (Euhe) by the main components 
Tabela 2. Massa seca aérea ( $\mathrm{kg} \mathrm{ha}^{-1}$ ) e contribuição no total (\%) das espécies presentes nos sistemas de produção avaliados na safra 2020/21

Table 2. Aerial dry matter $\left(\mathrm{kg} \mathrm{ha}^{-1}\right)$ and contribution to the total (\%) of species present in the production systems evaluated in the $2020 / 21$ cycle

\begin{tabular}{|c|c|c|c|c|c|c|c|c|c|c|}
\hline \multicolumn{2}{|l|}{ Espécies } & \multicolumn{2}{|c|}{ Lavoura } & \multicolumn{2}{|c|}{ Lavoura-Pecuária } & \multicolumn{2}{|c|}{ Lavoura-Floresta } & \multicolumn{2}{|c|}{$\begin{array}{c}\text { Lavoura- } \\
\text { Pecuária-Floresta }\end{array}$} & \multirow{2}{*}{$\frac{\mathrm{CV}}{\%}$} \\
\hline $\begin{array}{l}\text { Nome } \\
\text { Comum }\end{array}$ & Nome Científico & $\mathrm{Kg} \mathrm{ha}^{-1}$ & $\%$ & $\mathrm{Kg} \mathrm{ha}^{-1}$ & $\%$ & $\mathrm{Kg} \mathrm{ha}^{-1}$ & $\%$ & $\mathrm{Kg} \mathrm{ha}^{-1}$ & $\%$ & \\
\hline Capim Aries & M. maximus & 1194 & 62,4 & 1983 & 87,8 & 1049 & 17,9 & 7242 & 91,3 & 102,7 \\
\hline $\begin{array}{l}\text { Corda-de- } \\
\text { viola }\end{array}$ & Ipomoea sp. & 251 & 13,1 & 0 & 0 & 269 & 4,6 & 536 & 6,8 & 83 \\
\hline Assa-peixe & $\begin{array}{l}\text { Vernonia } \\
\text { polysphaera Baker }\end{array}$ & 14 & 0,7 & 164 & 7,3 & 5 & 0,1 & 87 & 1,1 & 109,7 \\
\hline Carrapichão & $\begin{array}{l}\text { Xanthium } \\
\text { strumarium L. }\end{array}$ & 88 & 4,6 & 0 & 0 & 0 & 0 & 35 & 0,4 & 135,2 \\
\hline Picão-preto & Bidens pilosa $\mathrm{L}$. & 274 & 14,3 & 111 & 4,9 & 26 & 0,4 & 21 & 0,3 & 109,4 \\
\hline Braquiária & U. brizantha & 57 & 3 & 0 & 0 & 2416 & 41,2 & 12 & 0,2 & 192,6 \\
\hline Hemártria & H. altíssima & 0 & 0 & 0 & 0 & 2101 & 35,8 & 0 & 0 & 200 \\
\hline \multirow[t]{2}{*}{ Leiteira } & $\begin{array}{l}\text { Euphorbia } \\
\text { heterophylla L. }\end{array}$ & 36 & 1,9 & 0 & 0 & 0 & 0 & 0 & 0 & 200 \\
\hline & Total & 1914 & & 2258 & & 5866 & & 7933 & & \\
\hline
\end{tabular}

CONAB. Acompanhamento da Safra Brasileira. Séries históricas. Disponível em: https://www.conab.gov.br/info-agro/safras/ serie-historica-das-safras. Acesso em: 10 outubro 2021.

COSER, A.C.; NASCIMENTO JUNIOR, D.; GOMIDE, J. A.; SILVA, J. F. C.; SILVA, M. A. GARCIA, R.; MARTINS, C. E. Utilização do Botanal em comparação a outros métodos de avaliação. Pesquisa Agropecuária Brasileira. Brasília, p.759-767, 1991.

DOMINSCHEK R., BARROSO A. A. M., LANG C. R., DE MORAES A., SULC R. M., SCHUSTER M. Z. Crop rotations with temporary grassland shifts weed patterns and allows herbicide-free management without crop yield loss. Journal of Cleaner Production, v.306, 2021, DOI: https://doi.org/10.1016/j. jclepro.2021.127140.

DOMINSCHEK, R.; KRUCHELSKI, S.; DEISS, L.; PORTUGAL, T.B.; DENARDIN, L.G.; MARTINS, A.P.; LANG, C.R.; DE MORAES, A. Sistemas integrados de produção agropecuária na promoção da intensificação sustentável: Boletim Técnico do NITA [recurso eletrônico]. Curitiba. Universidade Federal do Paraná, Departamento de Fitotecnia e Fitossanitarismo, 2018.

EMBRAPA. Resistência de plantas daninhas a herbicidas: conceitos, origem e evolução. Passo Fundo: Embrapa Trigo, 2006. 22p. ISSN: 1518-6512. Publicação Online. Disponível em: http://ainfo.cnptia.embrapa.
br/digital/bitstream/CNPT-2010/40676/1/pdo58.pdf. Acesso em: 25 julho 2021.

EMBRAPA. Sistema Brasileiro de Classificação de Solos. 5. ed., rev. e ampl. Brasília, DF: Embrapa, 2018. 356 p.

HARGREAVES, J.; KERR, J. BOTANAL - A comprehensive sampling and computing procedure for estimating pasture yield and composition. Computational package. St Lucia, Qld.: CSIRO Division of Tropical Crops and Pastures,1992.

IDR-PARANÁ. Instituto de Desenvolvimento Rural do Paraná - IAPAR-EMATER. Atlas Climático do Estado do Paraná [recurso eletrônico]. Londrina (PR): Instituto Agronômico do Paraná, 2019. 210p. Disponível em: http://www.idrparana.pr.gov.br/system/ files/publico/agrometeorologia/atlas-climatico/atlas-climatico-do-parana-2019.pdf. Acesso em: 03 abril 2021.

LEVINSKI-HUF, F. Adubação de sistemas e comportamento dos nutrientes em sistema de integração lavoura-pecuária. Tese (Doutorado), Universidade Tecnológica Federal do Paraná. Programa de Pós-Graduação em Agronomia. Pato Branco, PR. 2018.

PIASSETTA, R. R. L.; SOUZA, N.J.; MIKOS, A.P.; AUER, C.G. Legislação restritiva referente ao uso de agrotóxicos em municípios do Estado do Paraná. BIOFIX Scientific Journal, v.6, n.1, p.75-83, 2021. DOI: https://doi. org/10.5380/biofix.v6i1.77378.
PITELLI, R.A. Planta Daninha, ViçosaMG, v.33, n.3, 2015. DOI: https://doi. org/10.1590/S0100-83582015000300025.

RODRIGUES, V.N.; VON PINHO, R.G.; PAGLIS, C.M.; BUENO FILHO, J.S.S.; BRITO, A. $H$. Comparação entre métodos para estimar a produtividade de grãos de milho. Ciência e Agrotecnologia, v.29, n.1, p.34-42, 2005. DOI: https://doi.org/10.1590/S141370542005000100004

SCHUSTER, M.Z, PELISSARI, A.; DE MORAES, A.; HARRISON, S.K.; SULC, R.M.; LUSTOSA, S.B.C.; ANGHINONI, I.; CARVALHO, P.C.F. Grazing intensities affect weed seedling emergence and the seed bank in an integrated crop-livestock system. Agriculture, Ecosystems \& Environment, v.232, 2016, p.232-239. DOI: https://doi.org/10.1016/j. agee.2016.08.005.

SCHUSTER, M.Z.; LUSTOSA, S.B.C.; PELISSARI, A.; HARRISON, S.K.; SULC, R. M.; DEISS, L.; LANG, C.R.; CARVALHO, P.C.D.F.; GAZZIERO, D.L.P.; MORAES, A. Optimizing forage allowance for productivity and weed management in integrated crop-livestock systems. Agronomy for sustainable development, v.39, n.2, p.1-10, 2019. DOI: https://doi. org/10.1007/s13593-019-0564-4.

WEISBERGER D., NICHOLS V., LIEBMAN M. Does diversifying crop rotations suppress weeds? A meta-analysis. PloS ONE, v.14, n.7, p.e0219847, 2019. DOI: https:// doi.org/10.1371/journal.pone.0219847 